\title{
A STUDY OF NON-ASSOCIATIVE ORDERED SEMIGROUPS IN TERMS OF SEMILATTICES VIA SMALLEST (DOUBLE-FRAMED SOFT) IDEALS
}

\section{FAISAL YOUSUFZAI ${ }^{1}$, TAUSEEF ASIF $^{2}$, ASGHAR KHAN ${ }^{2, *}$ AND BIJAN DAVVAZ $^{3}$}

${ }^{1}$ Military College of Engineering National University of Sciences and Technology (NUST), Islamabad, Pakistan

${ }^{2}$ Department of Mathematics, Abdul Wali Khan University, Mardan, KPK, Pakistan

${ }^{3}$ Department of Mathematics, Yazd University, Yazd, Iran

*Corresponding author: asghar@awkum.edu.pk

\begin{abstract}
Soft set theory, introduced by Molodtsov has been considered as a successful mathematical tool for modeling uncertainties. A double-framed soft set is a generalization of a soft set, consisting of union soft sets and intersectional soft sets. An ordered $\mathcal{A G}$-groupoid can be referred to as a non-associative ordered semigroup, as the main difference between an ordered semigroup and an ordered $\mathcal{A} \mathcal{G}$-groupoid is the switching of an associative law. In this paper, we define the smallest left (right) ideals in an ordered $\mathcal{A G}$-groupoid and use them to characterize a strongly regular class of a unitary ordered $\mathcal{A G}$-groupoid along with its semilattices and double-framed soft (briefly DFS) $l$-ideals $(r$-ideals). We also give the concept of an ordered $\mathcal{A}^{*} \mathcal{G}^{* *}$-groupoid and investigate its structural properties by using the generated ideals and DFS $l$-ideals $(r$-ideals). These concepts will verify the existing characterizations and will help in achieving more generalized results in future works.
\end{abstract}

Received 2018-01-16; accepted 2018-04-06; published 2018-07-02.

2010 Mathematics Subject Classification. 00A00.

Key words and phrases. DFS-sets; ordered $\mathcal{A G}$-groupoid, pseudo-inverses; ordered $\mathcal{A}^{*} \mathcal{G}^{* *}$-groupoid, left invertive law; smallest ideals and DFS ideals.

(C) 2018 Authors retain the copyrights of their papers, and all open access articles are distributed under the terms of the Creative Commons Attribution License. 


\section{INTRODUCTION}

The concept of soft set theory was introduced by Molodtsov in [17]. This theory can be used as a generic mathematical tool for dealing with uncertainties. In soft set theory, the problem of setting the membership function does not arise, which makes the theory easily applied to many different fields [1,2,5-9]. At present, the research work on soft set theory in algebraic fields is progressing rapidly [20, 22-24]. A soft set is a parameterized family of subsets of the universe set. In the real world, the parameters of this family arise from the view point of fuzzy set theory. Most of the researchers of algebraic structures have worked on the fuzzy aspect of soft sets. Soft set theory is applied in the field of optimization by Kovkov in [13]. Several similarity measures have been discussed in [16], decision making problems have been studied in [22], reduction of fuzzy soft sets and its applications in decision making problems have been analyzed in [14]. The notions of soft numbers, soft derivatives, soft integrals and many more have been formulated in [15]. This concept have been used for forecasting the export and import volumes in international trade [26].

Recently, Jun et al. further extended the notion of softs set into double-framed soft sets and defined double-framed soft subalgebra of BCK/BCI algebra and studied the related properties in [8]. Jun et al. also defined the concept of a double-framed soft ideal (briefly, DFS ideal) of a BCK/BCI-algebra and gave many valuable results for this theory. In [12], Khan et al. have applied the idea of double-framed soft set to ordered semigroups and defined prime and irreducible DFS ideals of an ordered semigroup over a universe set $U$. Khan et al. have also characterized different classes of an ordered semigroup by using different $D F S$ ideals.

In the present paper, we apply the idea given by Jun et al. in [8], to ordered $\mathcal{A} \mathcal{G}$-groupoids. We introduce and investigate the notions of DFS l-ideals and DFS r-ideals, and study the relationship between these $D F S$ ideals in detail. As an application of our results we get characterizations of a strongly regular class of a unitary ordered $\mathcal{A G}$-groupoid (an ordered $\mathcal{A}^{*} \mathcal{G}^{* *}$-groupoid) in terms of its semilattices, one-sided (two-sided) ideals based on $D F S$-sets and generated commutative monoids.

\section{Preliminaries}

An $\mathcal{A G}$-groupoid is a non-associative and a non-commutative algebraic structure lying in a grey area between a groupoid and a commutative semigroup. Commutative law is given by $a b c=c b a$ in ternary operations. By putting brackets on the left of this equation, i.e. $(a b) c=(c b) a$, in 1972, M. A. Kazim and M. Naseeruddin introduced a new algebraic structure called a left almost semigroup abbreviated as an $\mathcal{L} \mathcal{A}$-semigroup [10]. This identity is called the left invertive law. P. V. Protic and N. Stevanovic called the same structure an Abel-Grassmann's groupoid abbreviated as an AG-groupoid [21].

This structure is closely related to a commutative semigroup because a commutative $\mathcal{A} \mathcal{G}$-groupoid is a semigroup [18]. It was proved in [10] that an $\mathcal{A G}$-groupoid $S$ is medial, that is, $a b \cdot c d=a c \cdot b d$ holds for all 
$a, b, c, d \in S$. An $\mathcal{A G}$-groupoid may or may not contain a left identity. The left identity of an $\mathcal{A}$-groupoid permits the inverses of elements in the structure. If an $\mathcal{A G}$-groupoid contains a left identity, then this left identity is unique [18]. In an $\mathcal{A G}$-groupoid $S$ with left identity, the paramedial law $a b \cdot c d=d c \cdot b a$ holds for all $a, b, c, d \in S$. By using medial law with left identity, we get $a \cdot b c=b \cdot a c$ for all $a, b, c \in S$. We should genuinely acknowledge that much of the ground work has been done by M. A. Kazim, M. Naseeruddin, Q. Mushtaq, M. S. Kamran, P. V. Protic, N. Stevanovic, M. Khan, W. A. Dudek and R. S. Gigon. One can be referred to $[3,4,11,18,19,21,25]$ in this regard.

An $\mathcal{A G}$-groupoid $(S, \cdot)$ together with a partial order $\leq$ on $S$ that is compatible with an $\mathcal{A G}$-groupoid operation, meaning that for $x, y, z \in S, x \leq y \Rightarrow z x \leq z y$ and $x z \leq y z$, is called an ordered $\mathcal{A G}$-groupoid [28].

Let us define a binary operation " $e_{e}$ (e-sandwich operation) on an ordered $\mathcal{A G}$-groupoid $(S, \cdot, \leq)$ with left identity e as follows:

$$
a \circ_{e} b=a e \cdot b, \forall a, b \in S
$$

Then $\left(\mathcal{S}, \circ_{e}, \leq\right)$ becomes an ordered semigroup [28].

Note that an ordered $\mathcal{A G}$-groupoid is the generalization of an ordered semigroup because if an ordered $\mathcal{A G}$-groupoid has a right identity then it becomes an ordered semigroup.

Let $\emptyset \neq A \subseteq S$, we denote $(A]$ by $(A]:=\{x \in S / x \leq a$ for some $a \in A\}$. If $A=\{a\}$, then we write $(\{a\}]$. For $\emptyset \neq A, B \subseteq S$, we denote $A B=:\{a b / a \in A, b \in B\}$.

- Let $S$ be an ordered $\mathcal{A G}$-groupoid. By an ordered $\mathcal{A G}$-subgroupoid of $S$, we means a nonempty subset $A$ of $S$ such that $\left(A^{2}\right] \subseteq A$.

- A nonempty subset $A$ of an ordered $\mathcal{A G}$-groupoid $S$ is called a left (right) ideal of $S$ if:

(i) $S A \subseteq A(A S \subseteq A)$;

(ii) if $a \in A$ and $b \in S$ such that $b \leq a$, then $b \in A$.

Equivalently: A nonempty subset $A$ of an ordered $\mathcal{A G}$-groupoid $S$ is called a left (right) ideal of $S$ if $(S A] \subseteq A((A S] \subseteq A)$.

- By two-sided ideal or simply ideal, we mean a nonempty subset of an ordered $\mathcal{A G}$-groupoid $S$ which is both left and right ideal of $S$.

Lemma 2.1. [28] Let $S$ be an ordered $\mathcal{A G}$-groupoid and $\emptyset \neq A, B \subseteq S$. Then the following hold:

(i) $A \subseteq(A]$;

(ii) If $A \subseteq B$, then $(A] \subseteq(B]$;

(iii) $(A](B] \subseteq(A B]$;

(iv) $(A]=((A]]$;

(vi) $((A](B]]=(A B]$;

(vii) $(T]=T$, for every ideal $T$ of $S$; 
(viii) $(S S]=S=S S$, if $S$ has a left identity.

\section{Soft Sets}

In [24], Sezgin and Atagun introduced some new operations on soft set theory and defined soft sets in the following way.

Let $U$ be an initial universe set, $E$ a set of parameters, $P(U)$ the power set of $U$ and $A \subseteq E$. Then a soft set $f_{A}$ over $U$ is a function defined by:

$$
f_{A}: E \rightarrow P(U) \text { such that } f_{A}(x)=\emptyset \text {, if } x \notin A .
$$

Here $f_{A}$ is called an approximate function. A soft set over $U$ can be represented by the set of ordered pairs

$$
f_{A}=\left\{\left(x, f_{A}(x)\right): x \in E, f_{A}(x) \in P(U)\right\}
$$

It is clear that a soft set is a parameterized family of subsets of $U$. The set of all soft sets is denoted by $S(U)$.

- Let $f_{A}, f_{B} \in S(U)$. Then $f_{A}$ is a soft subset of $f_{B}$, denoted by $f_{A} \simeq f_{B}$ if $f_{A}(x) \subseteq f_{B}(x)$ for all $x \in S$. Two soft sets $f_{A}, f_{B}$ are said to be equal soft sets if $f_{A} \simeq f_{B}$ and $f_{B} \simeq f_{A}$ and is denoted by $f_{A} \cong f_{B}$. The union of $f_{A}$ and $f_{B}$, denoted by $f_{A} \tilde{\cup} f_{B}$, is defined by $f_{A} \tilde{\cup} f_{B}=f_{A \cup B}$, where $f_{A \cup B}(x)=f_{A}(x) \cup f_{B}(x), \forall$ $x \in E$. In a similar way, we can define the intersection of $f_{A}$ and $f_{B}$.

- Let $S$ be an ordered $\mathcal{A G}$-groupoid, let $f_{A}, f_{B} \in S(U)$. Then the soft product [24] of $f_{A}$ and $f_{B}$, denoted by $f_{A} \sim f_{B}$, is defined as follows:

$$
\left(f_{A} \sim f_{B}\right)(x)= \begin{cases}\bigcup_{(y, z) \in A_{x}}\left\{f_{A}(y) \cap g_{B}(z)\right\} & \text { if } A_{x} \neq \emptyset \\ \emptyset & \text { if } A_{x}=\emptyset\end{cases}
$$

where $A_{x}=\{(y, z) \in S \times S / x \leq y z\}$.

- A double-framed soft pair $\left\langle\left(f_{A}^{+}, f_{A}^{-} ; A\right\rangle\right.$ is called a double-framed soft set (briefly, DFS-set of $A$ ) [8] of $A$ over $U$, where $f_{A}^{+}$and $f_{A}^{-}$are mappings from $A$ to $P(U)$. The set of all $D F S$-sets of $A$ over $U$ will be denoted by $D F S(U)$.

- Let $f_{A}=\left\langle\left(f_{A}^{+}, f_{A}^{-}\right) ; A\right\rangle$ and $g_{A}=\left\langle\left(g_{A}^{+}, g_{A}^{-}\right) ; A\right\rangle$ be two double-framed soft sets of an ordered $\mathcal{A G}$ groupoid $S$ over $U$. Then the uni-int soft product [12], denoted by $f_{A} \diamond g_{A}=\left\langle\left(f_{A}^{+} \widetilde{\circ} g_{A}^{+}, f_{A}^{-} \approx g_{A}^{-}\right) ; A\right\rangle$ is defined to be a double-framed soft set of $S$ over $U$, in which $f_{A}^{+} \widetilde{\circ} g_{A}^{+}$and $f_{A}^{-} \approx g_{A}^{-}$are mapping from $S$ to $P(U)$, given as follows: 


$$
\begin{aligned}
& f_{A}^{+} \tilde{\circ} g_{A}^{+}: S \longrightarrow P(U), x \longmapsto \begin{cases}\bigcup_{(y, z) \in A_{x}}\left\{f_{A}^{+}(y) \cap g_{A}^{+}(z)\right\} & \text { if } A_{x} \neq \emptyset \\
\emptyset & \text { if } A_{x}=\emptyset,\end{cases} \\
& f_{A}^{-} \tilde{\star} g_{A}^{-}: S \longrightarrow P(U), x \longmapsto \begin{cases}\bigcap_{(y, z) \in A_{x}}\left\{f_{A}^{-}(y) \cup g_{A}^{-}(z)\right\} & \text { if } A_{x} \neq \emptyset \\
U & \text { if } A_{x}=\emptyset .\end{cases}
\end{aligned}
$$

- Let $f_{A}=\left\langle\left(f_{A}^{+}, f_{A}^{-}\right) ; A\right\rangle$ and $g_{A}=\left\langle\left(g_{A}^{+}, g_{A}^{-}\right) ; A\right\rangle$ be two double-framed soft sets over a common universe set $U$. Then $\left\langle\left(f_{A}^{+}, f_{A}^{-}\right) ; A\right\rangle$ is called a double-framed soft subset (briefly, DFS-subset) [12] of $\left\langle\left(g_{A}^{+}, g_{A}^{-}\right) ; A\right\rangle$, denote by $\left\langle\left(f_{A}^{+}, f_{A}^{-}\right) ; A\right\rangle \sqsubseteq\left\langle\left(g_{A}^{+}, g_{A}^{-}\right) ; A\right\rangle$ if:

(i) $A \subseteq B$;

(ii) $(\forall e \in A)\left(\begin{array}{l}f_{A}^{+} \text {and } g_{A}^{+} \text {are identical approximations }\left(f_{A}^{+}(e) \subseteq g_{A}^{+}(e)\right) \\ f_{A}^{-} \text {and } g_{A}^{-} \text {are identical approximations }\left(f_{A}^{-}(e) \supseteq g_{A}^{-}(e)\right)\end{array}\right)$.

- For two $D F S$-sets $f_{A}=\left\langle\left(f_{A}^{+}, f_{A}^{-}\right) ; A\right\rangle$ and $g_{A}=\left\langle\left(g_{A}^{+}, g_{A}^{-}\right) ; A\right\rangle$ over $U$ are said to be equal, denoted by $\left\langle\left(f_{A}^{+}, f_{A}^{-}\right) ; A\right\rangle=\left\langle\left(g_{A}^{+}, g_{A}^{-}\right) ; A\right\rangle$, if $\left\langle\left(f_{A}^{+}, f_{A}^{-}\right) ; A\right\rangle \sqsubseteq\left\langle\left(g_{A}^{+}, g_{A}^{-}\right) ; A\right\rangle$ and $\left\langle\left(g_{A}^{+}, g_{A}^{-}\right) ; A\right\rangle \sqsubseteq\left\langle\left(f_{A}^{+}, f_{A}^{-}\right) ; A\right\rangle$.

- For two $D F S$-sets $f_{A}=\left\langle\left(f_{A}^{+}, f_{A}^{-}\right) ; A\right\rangle$ and $g_{A}=\left\langle\left(g_{A}^{+}, g_{A}^{-}\right) ; A\right\rangle$ over $U$, the DFS int-uni set [12] of $\left\langle\left(f_{A}^{+}, f_{A}^{-}\right) ; A\right\rangle$ and $\left\langle\left(g_{A}^{+}, g_{A}^{-}\right) ; A\right\rangle$, is defined to be a $D F S$-set $\left\langle\left(f_{A}^{+} \cap g_{A}^{+}, f_{A}^{-} \cup g_{A}^{-}\right) ; A\right\rangle$, where $f_{A}^{+} \cap g_{A}^{+}$and $f_{A}^{-} \cup g_{A}^{-}$are mapping given as follows:

$$
\begin{aligned}
& f_{A}^{+} \cap g_{A}^{+} \quad: \quad A \longrightarrow P(U), x \longmapsto f_{A}^{+}(x) \cap g_{A}^{+}(x) ; \\
& f_{A}^{-} \cup g_{A}^{-} \quad: \quad A \longrightarrow P(U), x \longmapsto f_{A}^{-}(x) \cup g_{A}^{-}(x) .
\end{aligned}
$$

It is denoted by $\left\langle\left(f_{A}^{+}, f_{A}^{-}\right) ; A\right\rangle \sqcap\left\langle\left(g_{A}^{+}, g_{A}^{-}\right) ; A\right\rangle=\left\langle\left(f_{A}^{+} \cap g_{A}^{+}, f_{A}^{-} \cup g_{A}^{-}\right) ; A\right\rangle$.

- A double-framed soft set $f_{A}=\left\langle\left(f_{A}^{+}, f_{A}^{-}\right) ; A\right\rangle$ of $S$ over $U$ is called a double-framed soft $\mathcal{A} \mathcal{G}$-subgroupoid (briefly, DFS $\mathcal{A} \mathcal{G}$-subgroupoid) of $S$ over $U$ if it satisfies $f_{A}^{+}(x y) \supseteq f_{A}^{+}(x) \cap f_{A}^{+}(y), f_{A}^{-}(x y) \subseteq f_{A}^{-}(x) \cup f_{A}^{-}(y)$, $\forall x, y \in S$.

- A double-framed soft set $f_{A}=\left\langle\left(f_{A}^{+}, f_{A}^{-}\right) ; A\right\rangle$ of $S$ over $U$ is called

(i) a double-framed soft left ideal (briefly, DFS l-ideal) of $S$ over $U$ if it satisfies:

(a) $f_{A}^{+}(x y) \supseteq f_{A}^{+}(y)$ and $f_{A}^{-}(x y) \subseteq f_{A}^{-}(y)$;

(b) $x \leq y \Longrightarrow f_{A}^{+}(x) \supseteq f_{A}^{+}(y)$ and $f_{A}^{-}(x) \subseteq f_{A}^{-}(y), \forall x, y \in S$.

(ii) a double-framed soft right ideal (briefly, DFS r-ideal) of $S$ over $U$ if it satisfies:

(a) $f_{A}^{+}(x y) \supseteq f_{A}^{+}(x)$ and $f_{A}^{-}(x y) \subseteq f_{A}^{-}(x)$;

(b) $x \leq y \Longrightarrow f_{A}^{+}(x) \supseteq f_{A}^{+}(y)$ and $f_{A}^{-}(x) \subseteq f_{A}^{-}(y), \forall x, y \in S$.

(iii) a double-framed soft ideal (briefly, DFS ideal) of $S$ over $U$, if it is both DFS l-ideal and DFS r-ideal of $S$ over $U$.

- Let $A$ be a nonempty subset of $S$. Then the characteristic double-framed soft mapping of $A$, denoted by $\left\langle\left(\mathcal{X}_{A}^{+}, \mathcal{X}_{A}^{-}\right) ; A\right\rangle=\mathcal{X}_{A}$ is defined to be a double-framed soft set, in which $\mathcal{X}_{A}^{+}$and $\mathcal{X}_{A}^{-}$are soft mappings 
over $U$, given as follows:

$$
\begin{aligned}
& \mathcal{X}_{A}^{+} \quad: \quad S \longrightarrow P(U), x \longmapsto\left\{\begin{array}{cc}
U & \text { if } x \in A \\
\emptyset & \text { if } x \notin A,
\end{array}\right. \\
& \mathcal{X}_{A}^{-} \quad: \quad S \longrightarrow P(U), x \longmapsto\left\{\begin{array}{cc}
\emptyset & \text { if } x \in A \\
U & \text { if } x \notin A .
\end{array}\right.
\end{aligned}
$$

Note that the characteristic mapping of the whole set $S$, denoted by $\mathcal{X}_{S}=\left\langle\left(\mathcal{X}_{S}^{+}, \mathcal{X}_{S}^{-}\right) ; S\right\rangle$, is called the identity double-framed soft mapping, where $\mathcal{X}_{S}^{+}(x)=U$ and $\mathcal{X}_{S}^{-}(x)=\emptyset, \forall x \in S$.

The following result holds for an ordered semigroup [6] just because of the closure property which makes very clear for an ordered $\mathcal{A G}$-groupoid to hold the same Lemma.

Lemma 3.1. For a nonempty subset $A$ of an ordered $\mathcal{A G}$-groupoid $S$, the following conditions are equivalent:

(i) $A$ is a left ideal (right ideal) of $S$;

(ii) The DFS set $\mathcal{X}_{A}$ of $S$ over $U$ is a DFS l-ideal (DFS r-ideal) of $S$ over $U$.

The following result holds for an ordered semigroup [12] just because of the closure property which makes very clear for an ordered $\mathcal{A G}$-groupoid to hold the same Lemma.

Lemma 3.2. Let $f_{A}=\left\langle\left(f_{A}^{+}, f_{A}^{-}\right) ; A\right\rangle$ be any DFS-set of an ordered $\mathcal{A G}$-groupoid $S$ over $U$. Then $f_{A}$ is a $D F S$-ideal (l-ideal) of $S$ over $U$ if and only if $f_{A} \diamond \mathcal{X}_{S} \sqsubseteq f_{A}\left(\mathcal{X}_{S} \diamond f_{A} \sqsubseteq f_{A}\right)$.

- A double-framed soft set $f_{A}=\left\langle\left(f_{A}^{+}, f_{A}^{-}\right) ; A\right\rangle$ of $S$ over $U$ is called DFS semiprime if $f_{A}(x) \sqsupseteq f_{A}\left(x^{2}\right)$, $\forall x \in A$.

Lemma 3.3. Let $A$ be any right (left) ideal of an ordered $\mathcal{A} \mathcal{G}$-groupoid $S$. Then $A$ is semiprime if and only if $\mathcal{X}_{A}$ is DFS semiprime.

Proof. Let $A$ be a right (left) ideal of $S$, then by Lemma 3.1, $\mathcal{X}_{A}$ is a $D F S r$-ideal (DFS l-ideal) of $S$ over $U$. Let $a^{2} \in A$, then $\mathcal{X}_{A}^{+}(a) \supseteq \mathcal{X}_{A}^{+}\left(a^{2}\right)$, therefore $\mathcal{X}_{A}^{+}\left(a^{2}\right)=U \subseteq \mathcal{X}_{A}^{+}(a)$, this implies $\mathcal{X}_{A}^{+}(a)=U$ and similarly $\mathcal{X}_{A}^{-}(a)=\emptyset$. Thus $a \in A$ and therefore $A$ is semiprime. Converse is simple.

Remark 3.1. The set $(D F S(U), \diamond, \sqsubseteq)$ forms an ordered $\mathcal{A G}$-groupoid and satisfies all the basic laws.

Remark 3.2. If $S$ is an ordered $\mathcal{A} \mathcal{G}$-groupoid, then $\mathcal{X}_{S} \diamond \mathcal{X}_{S}=\mathcal{X}_{S}$.

The following result also holds for an ordered semigroup [12] just because of the closure property which is very trivial for an ordered $\mathcal{A} \mathcal{G}$-groupoid to hold the same Lemma.

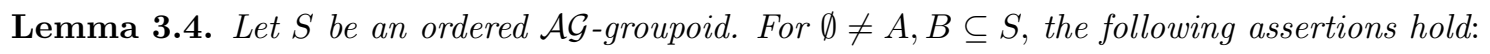


(i) $A \subseteq B \Leftrightarrow \mathcal{X}_{A} \sqsubseteq \mathcal{X}_{B}$;

(ii) $\mathcal{X}_{A} \sqcap \mathcal{X}_{B}=\mathcal{X}_{A \cap B}$;

(iii) $\mathcal{X}_{A} \sqcup \mathcal{X}_{B}=\mathcal{X}_{A \cup B}$;

(iv) $\mathcal{X}_{A} \diamond \mathcal{X}_{B}=\mathcal{X}_{(A B]}$.

\section{On DFS Strongly Regular ordered $\mathcal{A G}$-Groupoids}

Throughout this paper, let $E=S$, where $S$ is a unitary ordered $\mathcal{A G}$-groupoid, unless otherwise stated. By a unitary ordered $\mathcal{A G}$-groupoid, we shall mean an ordered $\mathcal{A G}$-groupoid with left identity.

4.1. Basic Results. This section contains some examples and basic results which will be essential for up coming section.

Theorem 4.1. Let $S$ be an ordered $\mathcal{A G}$-groupoid. A nonempty subset $A$ of $S$ is a left (resp. right) ideal of $S$ if and only if the DFS-set $\left\langle\left(g_{B}^{+}, g_{B}^{-}\right) ; B\right\rangle$, defined by

$$
g_{B}^{+}(x)=\left\{\begin{array}{c}
\gamma_{1} \text { if } x \in A \\
\gamma_{2} \text { if } x \in S \backslash A
\end{array}\right\} \text { and } g_{B}^{-}(x)=\left\{\begin{array}{c}
\delta_{1} \text { if } x \in A \\
\delta_{2} \text { if } x \in S \backslash A
\end{array}\right\},
$$

is a DFS l-ideal (resp. DFS $r$-ideal) of $S$ over $U$, where $\gamma_{1}, \gamma_{2}, \delta_{1}, \delta_{2} \subseteq U$ such that $\gamma_{2} \subseteq \gamma_{1}$ and $\delta_{1} \subseteq \delta_{2}$.

Proof. Necessity. Let $x, y \in S$ be such that $x \leq y$. If $y \notin A$, then $g_{B}^{+}(y)=\gamma_{2} \subseteq g_{B}^{+}(x)$ and $g_{B}^{-}(y)=\delta_{2} \supseteq$ $g_{B}^{-}(x)$. If $y \in A$, then $\gamma_{1}=g_{B}^{+}(y)$ and $\delta_{2}=g_{B}^{-}(y)$. Since $x \leq y \in A$, and $A$ is a left ideal of $S$, we have $x \in A$. Then $g_{B}^{+}(x)=\gamma_{1}=g_{B}^{+}(y)$ and $g_{B}^{-}(x)=\delta_{1}=g_{B}^{-}(y)$. For $x, y \in S$, we discuss the following two cases.

Case 1. If $x \in S$ and $y \in A$, then $x y \in A$ and we have $g_{B}^{+}(x)=\gamma_{1}=g_{B}^{+}(y)$ and $g_{B}^{-}(x)=\delta_{1}=g_{B}^{-}(y)$.

Case 2. If $x \in S$ and $y \notin A$, then $g_{B}^{+}(y)=\gamma_{2} \subseteq g_{B}^{+}(x y)$ and $g_{B}^{-}(y)=\delta_{2} \supseteq g_{B}^{-}(x y)$. Therefore $\left\langle\left(g_{B}^{+}, g_{B}^{-}\right) ; B\right\rangle$ is a $D F S l$-ideal of $S$ over $U$. Similarly we can prove the result for a $D F S r$-ideal of $S$ over $U$.

Sufficiency. Assume that $\left\langle\left(g_{B}^{+}, g_{B}^{-}\right) ; B\right\rangle$ is a DFS $l$-ideal of $S$ over $U$. Let $x, y \in S$ be such that $x \leq y$. If $y \in A$, then $g_{B}^{+}(y) \supseteq \gamma_{1}$ and $g_{B}^{-}(y) \subseteq \delta_{1}$. Since $g_{B}^{+}(x) \supseteq g_{B}^{+}(y) \supseteq \gamma_{1}$ and $g_{B}^{-}(x) \subseteq g_{B}^{+}(y) \subseteq \delta_{1}$, we have $x \in A$. Let $x \in S$ and $y \in A$, then $g_{B}^{+}(y)=\gamma_{1}$ and $g_{B}^{-}(y)=\delta_{1}$. By hypothesis, $g_{B}^{+}(x y) \supseteq g_{B}^{+}(y)=\gamma_{1}$ and $g_{B}^{-}(x y) \subseteq g_{B}^{+}(y)=\delta_{1}$. Hence $x y \in A$. Thus $A$ is a left ideal of $S$. Similarly, we can show that $A$ is a right ideal of $S$.

Example 4.1. There are six different chemicals which have been used in an experiment. Take a collection of chemicals as the initial universe set $U$ given by

$$
U=\left\{\gamma_{1}, \gamma_{2}, \gamma_{3}, \gamma_{4}, \gamma_{5}, \gamma_{6}\right\}
$$

Let a set of parameters $E=\{1,2,3,4,5\}$ be a set of particular properties of each chemical in $U$ with the following type of natures: 
1 stands for the parameter "density",

2 stands for the parameter "melting point",

3 stands for the parameter "combustion",

4 stands for the parameter "enthalpy",

5 stands for the parameter "toxicity".

Let us define the following binary operation and order on a set of parameters $E$ as follows.

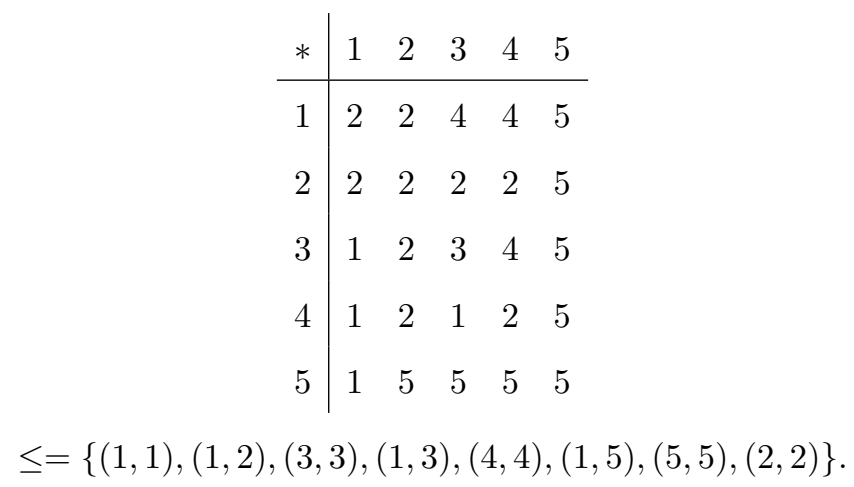

It is easy to observe that $(E, *, \leq)$ is a unitary ordered $\mathcal{A G}$-groupoid.

Let $A=\{1,2,5\}$ and define a $D F S$-set $\left\langle\left(f_{A}^{+}, f_{A}^{-}\right) ; A\right\rangle$ of $S$ over $U$ as follows:

$$
f_{A}^{+}(x)=\left\{\begin{array}{c}
\left\{\gamma_{1}, \gamma_{2}\right\} \text { if } x=1 \\
\left\{\gamma_{1}, \gamma_{2}, \gamma_{3}\right\} \text { if } x=2 \\
\left\{\gamma_{5}\right\} \text { if } x=3 \\
\left\{\gamma_{5}\right\} \text { if } x=4 \\
\left\{\gamma_{1}, \gamma_{2}, \gamma_{3}, \gamma_{4}\right\} \text { if } x=5
\end{array}\right\} \text { and } f_{A}^{-}(x)=\left\{\begin{array}{c}
\left\{\gamma_{1}, \gamma_{2}, \gamma_{3}\right\} \text { if } x=1 \\
\left\{\gamma_{1}, \gamma_{2}\right\} \text { if } x=2 \\
\{\} \text { if } x=3 \\
\{\} \text { if } x=4 \\
\left\{\gamma_{2}\right\} \text { if } x=5
\end{array}\right\} \text {. }
$$

Then it is easy to verify that $\left\langle\left(f_{A}^{+}, f_{A}^{-}\right) ; A\right\rangle$ is a DFS l-ideal of $S$ over $U$.

Let $B=\{1,2,4\}$ and define a $D F S$-set $\left\langle\left(g_{B}^{+}, g_{B}^{-}\right) ; B\right\rangle$ of $S$ over $U$ as follows:

$$
g_{B}^{+}(x)=\left\{\begin{array}{c}
\left\{\gamma_{4}\right\} \text { if } x=1 \\
\left\{\gamma_{2}, \gamma_{3}, \gamma_{4}\right\} \text { if } x=2 \\
\left\{\gamma_{1}, \gamma_{2}, \gamma_{5}\right\} \text { if } x=3 \\
\left\{\gamma_{1}, \gamma_{2}, \gamma_{5}\right\} \text { if } x=4 \\
\left\{\gamma_{2}, \gamma_{4}\right\} \text { if } x=5
\end{array}\right\} \text { and } g_{B}^{-}(x)=\left\{\begin{array}{c}
\left\{\gamma_{1}, \gamma_{2}, \gamma_{3}, \gamma_{4}\right\} \text { if } x=1 \\
\left\{\gamma_{2}, \gamma_{3}\right\} \text { if } x=2 \\
\left\{\gamma_{1}, \gamma_{2}, \ldots, \gamma_{5}\right\} \text { if } x=3 \\
\left\{\gamma_{1}, \gamma_{2}, \gamma_{3}, \gamma_{4}\right\} \text { if } x=4 \\
\left\{\gamma_{2}, \gamma_{3}\right\} \text { if } x=5
\end{array}\right\} .
$$

Then it is easy to verify that $\left\langle\left(g_{B}^{+}, g_{B}^{-}\right) ; B\right\rangle$ is a $D F S$-ideal of $S$ over $U$.

Remark 4.1. Every DFS r-ideal of a unitary ordered $\mathcal{A G}$-groupoid $S$ over $U$ is a DFS l-ideal of $S$ over $U$ but the converse inclusion is not true in general which can be followed from above example. 
Lemma 4.1. Let $R$ be a right ideal and $L$ be a left ideal of a unitary ordered $\mathcal{A G}$-groupoid $S$. Then $(R L]$ is a left ideal of $S$.

Proof. Let $R$ be a right ideal and $L$ be a left ideal of $S$. Then by using Lemma 2.1, we get $S(R L]=$ $(S S](R L] \subseteq(S S \cdot R L]=(S R \cdot S L] \subseteq(S R \cdot(S L]]=(S R \cdot L]=((S S] R \cdot L] \subseteq((S S) R \cdot L]=((R S) S \cdot L] \subseteq$ $((R S] S \cdot L] \subseteq(R L]$, which shows that $(R L]$ is a left ideal of $S$.

Lemma 4.2. Let $S$ be a unitary ordered $\mathcal{A G}$-groupoid. If $a=a^{2}$ for all $a \in S$, then $R_{a}=\left(S a \cup S a^{2}\right]$ is the smallest right ideal of $S$ containing a.

Proof. Assume that $a=a^{2}$ for all $a \in S$. Then by using Lemma 2.1, we have

$$
\begin{aligned}
\left(S a \cup S a^{2}\right] S & =\left(S a \cup S a^{2}\right](S] \subseteq\left(\left(S a \cup S a^{2}\right) S\right]=\left(S a \cdot S \cup S a^{2} \cdot S\right] \\
& =\left(S a \cdot S S \cup S a^{2} \cdot S S\right]=\left(S \cdot a S \cup S \cdot a^{2} S\right]=\left(a \cdot S S \cup a^{2} \cdot S S\right] \\
& =\left(a^{2} \cdot S S \cup a^{2} \cdot S S\right]=\left(S S \cdot a^{2} \cup S S \cdot a^{2}\right]=\left(S a \cup S a^{2}\right],
\end{aligned}
$$

which shows that $\left(S a \cup S a^{2}\right]$ is a right ideal of $S$. It is easy to see that $a \in\left(S a \cup S a^{2}\right]$. Let $R$ be another right ideal of $S$ containing $a$. Since

$$
\left(S a \cup S a^{2}\right]=(S S \cdot a \cup a \cdot S a]=(a S \cdot S \cup a \cdot S a] \subseteq(R S \cdot S \cup R S] \subseteq R,
$$

Hence $\left(S a \cup S a^{2}\right]$ is the smallest right ideal of $S$ containing $a$.

Lemma 4.3. Let $S$ be a unitary ordered $\mathcal{A G}$-groupoid and $a=a^{2}$ for all $a \in S$. Then $S$ becomes a commutative monoid.

Proof. It is simple.

Corollary 4.1. $R_{a}=\left(S a \cup S a^{2}\right]$ is the smallest right ideal of an ordered commutative monoid $S$ containing $a$.

Lemma 4.4. Let $S$ be a unitary ordered $\mathcal{A G}$-groupoid and $a \in S$. Then $L_{a}=(S a]$ is the smallest left ideal of $S$ containing a.

Proof. It is simple.

Theorem 4.2. Let $S$ be a unitary ordered $\mathcal{A G}$-groupoid and $\emptyset \neq E \subseteq S$. Then the following assertions hold:

(i) E forms a semilattice, where $E=\left\{x \in S: x=x^{2}\right\}$;

(ii) $E$ is a singleton set, if $a=a x \cdot a, \forall a, x \in S$. 
Proof. (i). It is simple.

(ii). Let $y, z \in E$. Then by using $(i)$, we get

$$
y=y z \cdot y=z y \cdot y=y y \cdot z=y z=z y=z z \cdot y=y z \cdot z=z y \cdot z=z .
$$

- Recall that an ordered $\mathcal{A G}^{* *}$-groupoid is an ordered $\mathcal{A G}$-groupoid in which $a \cdot b c=b \cdot a c, \forall a, b, c \in S$. Note that an ordered $\mathcal{A G}^{* *}$-groupoid also satisfies the paramedial law as well.

Now let us introduce the concept of an ordered $\mathcal{A}^{*} \mathcal{G}^{* *}$-groupoid as follows:

- An ordered $\mathcal{A G}^{* *}$-groupoid $S$ is called an ordered $\mathcal{A}^{*} \mathcal{G}^{* *}$-groupoid if $S=\left(S^{2}\right]$.

Corollary 4.2. Let $S$ be an ordered $\mathcal{A}^{*} \mathcal{G}^{* *}$-groupoid and $\emptyset \neq E \subseteq S$. Then the following assertions hold:

(i) E forms a semilattice, where $E=\left\{x \in S: x=x^{2}\right\}$;

(ii) $E$ is a singleton set if $a=a x \cdot a, \forall a, x \in S$.

Lemma 4.5. Let $S$ be an ordered $\mathcal{A}^{*} \mathcal{G}^{* *}$-groupoid. Then $\langle R\rangle_{a^{2}}=\left(S a^{2} \cup a^{2}\right]\left(\langle L\rangle_{a}=(S a \cup a]\right)$ is the right (resp. left) ideal of $S$.

Proof. Let $a \in S$, then by using Lemma 2.1, we get

$$
\begin{aligned}
\left(S a^{2} \cup a^{2}\right] S & =\left(S a^{2} \cup a^{2}\right](S]=\left(\left(S a^{2} \cup a^{2}\right) S\right]=\left(S a^{2} \cdot S \cup a^{2} S\right] \\
& =\left(S S \cdot a^{2} S \cup S S \cdot a a\right]=\left(S \cdot a^{2} S \cup S a^{2}\right] \\
& =\left(a^{2} \cdot S S \cup S a^{2}\right]=\left(S a^{2}\right] \subseteq\left(S a^{2} \cup a^{2}\right]
\end{aligned}
$$

which is what we set out to prove. Similarly we can prove that $S(S a \cup a] \subseteq(S a \cup a]$.

- An element $a$ of an ordered $\mathcal{A G}$-groupoid $S$ is called a strongly regular element of $S$, if there exists some $x$ in $S$ such that $a \leq a x \cdot a$ and $a x=x a$, where $x$ is called a pseudo-inverse of a. $S$ is called strongly regular ordered $\mathcal{A G}$-groupoid if all elements of $S$ are strongly regular.

Theorem 4.3. Let $S$ be an ordered $\mathcal{A G}$-groupoid (an ordered $\mathcal{A}^{*} \mathcal{G}^{* *}$-groupoid) with left identity. An element $a$ of $S$ is strongly regular if and only if $a \leq a x \cdot$ ay for some $x, y \in S$.

Proof. Necessity. Let $a \in S$ is strongly regular, then $a \leq a x \cdot a \leq(a x) \cdot(x a)(a x \cdot a)=(a x) \cdot(a \cdot a x)(a x)=$ $(a x) \cdot a((a \cdot a x) x)=a x \cdot a y$, where $(a \cdot a x) x=y \in S$. Thus $a \leq a x \cdot a y$ for some $x, y \in S$.

Sufficiency. Let $a \in S$ such that $a \leq a x \cdot a y$ for some $x, y \in S$, then $a \leq a x \cdot a y=(a y \cdot x) a=(x y \cdot a) a=u a \cdot a$, where $x y=u \in S$. Thus $a u \leq(u a \cdot a) u=u a \cdot u a=u(u a \cdot a) \leq u a$, and $a \leq u a \cdot a=a u \cdot a$. Thus $S$ is strongly regular. 
Lemma 4.6. Let $f_{A}=\left\langle\left(f_{A}^{+}, f_{A}^{-}\right) ; A\right\rangle$ be any DFS r-ideal (DFS l-ideal) of a strongly regular ordered $\mathcal{A}^{*} \mathcal{G}^{* *}$-groupoid $S$ over $U$. Then the following assertions hold:

(i) $f_{A}=f_{A} \diamond \mathcal{X}_{S}\left(f_{A}=\mathcal{X}_{S} \diamond f_{A}\right)$;

(ii) $f_{A}$ is DFS semiprime.

Proof. It is simple.

4.2. Characterization Problems. In this section, we generalize the results of an ordered semigroup and get some interesting characterizations which we usually do not find in an ordered semigroup.

From now onward, $R$ (resp. $L$ ) will denote any right (resp. left) ideal of an ordered $\mathcal{A G}$-groupoid $S ; R_{a}$ (resp. $L_{a}$ ) will denote any smallest right (resp. smallest left) ideal of $S$ containing $a$. Any DFS $r$-ideal of an ordered $\mathcal{A G}$-groupoid $S$ (resp. DFS l-ideal of $S$ ) over $U$ will be denoted by $f_{A}$ (resp. $\left.g_{B}\right)$ unless otherwise specified.

Theorem 4.4. Let $f_{A}, g_{B}$ be any DFS l-ideals of a unitary ordered $\mathcal{A G}$-groupoid $S$. Then the following conditions are equivalent:

(i) $S$ is strongly regular;

(ii) $S$ is strongly regular commutative monoid;

(iii) $\left(R_{a} L_{a}\right] \cap L_{a}=\left(\left(R_{a} \cdot R_{a} L_{a}\right) L_{a} \cdot L_{a}\right],\left(a=a^{2}, \forall a \in S\right)$;

(iv) $(R L] \cap L=((R \cdot R L) L \cdot L]$;

(v) $f_{A} \sqcap g_{B}=\left(f_{A} \diamond g_{B}\right) \diamond f_{A}$;

(vi) $S$ is strongly regular and $|E|=1,(a=a x \cdot a, \forall a, x \in E)$;

(vii) $S$ is strongly regular and $\emptyset \neq E \subseteq S$ is semilattice.

Proof. $(i) \Longrightarrow$ (vii) : It can be followed from Theorem $4.2(i)$.

(vii) $\Longrightarrow(v i)$ : It can be followed from Theorem $4.2($ ii) .

$(v i) \Longrightarrow(v)$ : Let $f_{A}$ and $g_{B}$ be any DFS $l$-ideals of a strongly regular $S$ over $U$. Now for $a \in S$, there exist some $x, y \in S$ such that $a \leq a x \cdot a y=y a \cdot x a \leq y(a x \cdot a y) \cdot x a=(a x)(y \cdot a y) \cdot x a=(a y \cdot y)(x a) \cdot x a=\left(y^{2} a \cdot x a\right)(x a)$. Thus $\left(y^{2} a \cdot x a, x a\right) \in A_{a}$. Therefore

$$
\begin{aligned}
\left(\left(f_{A}^{+} \tilde{\circ} g_{B}^{+}\right) \widetilde{\circ} f_{A}^{+}\right)(a) & =\bigcup_{\left(y^{2} a \cdot x a, x a\right) \in A_{a}}\left\{\left(f_{A}^{+} \tilde{\circ} g_{B}^{+}\right)\left(y^{2} a \cdot x a\right) \cap f_{A}^{+}(x a)\right\} \\
& \supseteq \bigcup_{y^{2} a \cdot x a \leq y^{2} a \cdot x a}\left\{f_{A}^{+}\left(y^{2} a\right) \cap g_{B}^{+}(x a)\right\} \cap f_{A}^{+}(x a) \\
& \supseteq f_{A}^{+}\left(y^{2} a\right) \cap g_{B}^{+}(x a) \cap f_{A}^{+}(x a) \supseteq f_{A}^{+}(a) \cap g_{B}^{+}(a),
\end{aligned}
$$

and similarly, we get 


$$
\begin{aligned}
\left(\left(f_{A}^{-} \tilde{\star} g_{B}^{-}\right) \approx f_{A}^{-}\right)(a) & =\bigcap_{\left(y^{2} a \cdot x a, x a\right) \in A_{a}}\left\{\left(f_{A}^{-} \approx g_{B}^{-}\right)\left(y^{2} a \cdot x a\right) \cup f_{A}^{-}(x a)\right\} \\
& \subseteq \bigcap_{y^{2} a \cdot x a \leq y^{2} a \cdot x a}\left\{f_{A}^{-}\left(y^{2} a\right) \cup g_{B}^{-}(x a)\right\} \cup f_{A}^{-}(x a) \\
& \subseteq f_{A}^{-}\left(y^{2} a\right) \cup g_{B}^{-}(x a) \cup f_{A}^{-}(x a) \subseteq f_{A}^{-}(a) \cup g_{B}^{-}(a),
\end{aligned}
$$

which shows that $\left(f_{A} \diamond g_{B}\right) \diamond f_{A} \sqsupseteq f_{A} \sqcap g_{B}$. By using Lemmas 3.2 and 4.6, it is easy to show that $\left(f_{A} \diamond g_{B}\right) \diamond f_{A} \sqsubseteq f_{A} \sqcap g_{B}$. Thus $f_{A} \sqcap g_{B}=\left(f_{A} \diamond g_{B}\right) \diamond f_{A}$.

$(v) \Longrightarrow(i v)$ : Let $R$ and $L$ be any right and left ideals of $S$ respectively. Then by using Lemmas 3.1 and 4.1, $\mathcal{X}_{(R L]}$ and $\mathcal{X}_{L}$ are the DFS l-ideals of $S$ over $U$. Now by using Lemma 3.4, we get $\mathcal{X}_{(R L] \cap L}=$ $\mathcal{X}_{(R L]} \sqcap \mathcal{X}_{L}=\left(\mathcal{X}_{(R L]} \diamond \mathcal{X}_{L}\right) \diamond \mathcal{X}_{(R L]}=\mathcal{X}_{((R L] L \cdot(R L]]}$, which give us $(R L] \cap L=((R L] L \cdot(R L]]$. Now by using Lemma 2.1, we get

$$
\begin{aligned}
((R L] L \cdot(R L]] & =((R L) L \cdot R L]=\left(L^{2} R \cdot R L\right]=\left(L R \cdot R L^{2}\right] \\
& =\left(R\left(L R \cdot L^{2}\right)\right]=\left(R\left(L^{2} \cdot R L\right)\right]=\left(R\left(R \cdot L^{2} L\right)\right] \\
& =\left(R \cdot R L^{3}\right]=\left(R\left(R \cdot L^{2} L\right)\right]=\left(R\left(L^{2} \cdot R L\right)\right] \\
& =((R \cdot R L) L \cdot L] .
\end{aligned}
$$

$(i v) \Longrightarrow($ iii $)$ : It is simple.

$($ iii $) \Longrightarrow($ ii $)$ : Since $\left(S a \cup S a^{2}\right]$ is the smallest right ideal of $S$ containing $a$ and $(S a]$ is the smallest left ideal of $S$ containing $a$, where $a=a^{2}, \forall a \in S$. Thus by using given assumption and Lemma 2.1, we get

$$
\begin{aligned}
a & \in \quad\left(\left(S a \cup S a^{2}\right](S a]\right] \cap(S a]=\left(\left(\left(S a \cup S a^{2}\right] \cdot\left(S a \cup S a^{2}\right](S a]\right)(S a] \cdot(S a]\right] \\
& =\left(\left(\left(S a \cup S a^{2}\right) \cdot\left(S a \cup S a^{2}\right)(S a)\right)(S a) \cdot(S a)\right] \subseteq(S(S a) \cdot(S a)] \\
& =\left(S^{2} a \cdot S a\right]=(S a \cdot S a]=(a S \cdot a S] .
\end{aligned}
$$

Hence by using Lemma 4.2, $S$ is strongly regular commutative monoid.

$($ ii $) \Longrightarrow(i)$ : It is obvious.

Theorem 4.5. Let $S$ be an ordered $\mathcal{A G}$-groupoid. Then the following conditions are equivalent:

(i) $S$ is strongly regular;

(ii) $S$ is strongly regular commutative monoid;

(iii) $R_{a} \cap L_{a}=\left(R_{a}\left(L_{a} R_{a} \cdot R_{a}\right)\right],\left(a=a^{2}, \forall a \in S\right)$;

(iv) $R \cap L=(R(L R \cdot R)]$;

(v) $f_{A} \sqcap g_{B}=f_{A}^{3} \diamond g_{B}$; 
(vi) $S$ is strongly regular and $|E|=1,(a=a x \cdot a, \forall a, x \in E)$;

(vii) $S$ is strongly regular and $\emptyset \neq E \subseteq S$ is semilattice.

Proof. $(i) \Longrightarrow$ (vii) : It can be followed from Theorem $4.2(i)$.

$(v i i) \Longrightarrow(v i)$ : It can be followed from Theorem $4.2($ ii)

$(v i) \Longrightarrow(v)$ : Let $f_{A}$ and $g_{B}$ be any DFS r-ideal and DFS l-ideal of a strongly regular $S$ over $U$ respectively. From Lemma 3.2, it is easy to show that $f_{A}^{+3} \diamond g_{B}^{+} \sqsubseteq f_{A}^{+} \sqcap g_{B}^{+}$. Now for $a \in S$, there exist some $x, y \in S$ such that

$$
\begin{aligned}
a & \leq a x \cdot a y \leq(a x \cdot a y) x \cdot(a x \cdot a y) y=y(a x \cdot a y) \cdot x(a x \cdot a y) \\
& =(a x)(y \cdot a y) \cdot(a x)(x \cdot a y)=(a x)\left(a y^{2}\right) \cdot(a x)(a \cdot x y) \\
& =\left(y^{2} a\right)(x a) \cdot(a x)(a \cdot x y)=((a x)(a \cdot x y))(x a) \cdot y^{2} a \\
& =((a x)(a \cdot x y))(e x \cdot a) \cdot y^{2} a=((a x)(a \cdot x y))(a x \cdot e) \cdot y^{2} a \\
& =b c \cdot y^{2} a=d \cdot y^{2} a, \text { where } d=b c=((a x)(a \cdot x y))(a x \cdot e) .
\end{aligned}
$$

Thus

$$
\begin{aligned}
\left(\left(f_{A}^{+} \tilde{\circ} f_{A}^{+}\right) \widetilde{\circ} f_{A}^{+}\right)(d) & =\bigcup_{d \leq b c}\left\{\left(f_{A}^{+} \widetilde{\circ} f_{A}^{+}\right)(b) \cap f_{A}^{+}(c)\right\} \supseteq\left(f_{A}^{+} \tilde{\circ} f_{A}^{+}\right)(b) \cap f_{A}^{+}(c) \\
& =\bigcup_{b \leq(a x)(a \cdot x y)}\left\{f_{A}^{+}(a x) \cap f_{A}^{+}(a \cdot x y)\right\} \cap f_{A}^{+}(a x \cdot e) \\
& \supseteq f_{A}^{+}(a x) \cap f_{A}^{+}(a \cdot x y) \cap f_{A}^{+}(a x \cdot e) \supseteq f_{A}^{+}(a) .
\end{aligned}
$$

Therefore

$$
\left(f_{A}^{+3} \tilde{\circ} g_{B}^{+}\right)(a)=\bigcup_{a \leq d \cdot y^{2} a}\left\{\left(\left(f_{A}^{+} \tilde{\circ} f_{A}^{+}\right) \tilde{\circ} f_{A}^{+}\right)(d) \cap g_{B}^{+}\left(y^{2} a\right)\right\} \supseteq f_{A}^{+}(a) \cap g_{B}^{+}(a)
$$

which shows that $f_{A}^{+} \cap g_{B}^{+} \subseteq f_{A}^{+3} \tilde{\circ} g_{B}^{+}$, and similarly $f_{A}^{-} \cup g_{B}^{-} \supseteq f_{A}^{-3} \widetilde{\star} g_{B}^{-}$. Thus $f_{A} \sqcap g_{B}=f_{A}^{3} \diamond g_{B}$.

$(v) \Longrightarrow(i v)$ : Let $R$ and $L$ be any right and left ideals of $S$ respectively. Then by using Lemma 3.1, $\mathcal{X}_{R}$ and $\mathcal{X}_{L}$ are the DFS r-ideal and DFS $l$-ideal of $S$ over $U$ respectively. Now by using Lemma 3.4, we get

$$
\mathcal{X}_{R \cap L}=\mathcal{X}_{R} \sqcap \mathcal{X}_{L}=\left(\left(\mathcal{X}_{R} \diamond \mathcal{X}_{R}\right) \diamond \mathcal{X}_{R}\right) \diamond \mathcal{X}_{L}=\mathcal{X}_{\left(R^{3}\right]} \diamond \mathcal{X}_{L}=\mathcal{X}_{\left(\left(R^{3}\right] L\right]}
$$

which implies that $R \cap L=\left(\left(R^{3}\right] L\right]$. Now by using Lemma 2.1, we get $R \cap L=\left(\left(R^{3}\right] L\right]=\left(R^{3} L\right]=$ $\left(R^{2} R \cdot L\right]=\left(L R \cdot R^{2}\right]=\left(R^{2} \cdot R L\right]=\left(R \cdot R^{2} L\right]=(R(L R \cdot R)]$.

$(i v) \Longrightarrow(i i i)$ : It is simple. 
$(i i i) \Longrightarrow(i i)$ : Since $\left(S a \cup S a^{2}\right]$ is the smallest right ideal of $S$ containing $a$ and $(S a]$ is the smallest left ideal of $S$ containing $a$. Thus by using given assumption and Lemma 2.1, we get

$$
\begin{aligned}
a & \in\left(S a \cup S a^{2}\right] \cap(S a]=\left(\left(S a \cup S a^{2}\right]\left((S a]\left(S a \cup S a^{2}\right] \cdot\left(S a \cup S a^{2}\right]\right)\right] \\
& =\left(\left(S a \cup S a^{2}\right)\left((S a)\left(S a \cup S a^{2}\right) \cdot\left(S a \cup S a^{2}\right)\right)\right] \subseteq\left(S\left(S\left(S a \cup S a^{2}\right) \cdot\left(S a \cup S a^{2}\right)\right)\right] \\
& =\left(S\left(\left(S^{2} a \cup S^{2} a^{2}\right)\left(S a \cup S a^{2}\right)\right)\right]=\left(\left(S^{2} a \cup S^{2} a^{2}\right)\left(S\left(S a \cup S a^{2}\right)\right)\right] \\
& =\left(\left(S^{2} a \cup S^{2} a^{2}\right)\left(S^{2} a \cup S^{2} a^{2}\right)\right]=\left(\left(S a \cup a^{2} S^{2}\right)\left(S a \cup a^{2} S^{2}\right)\right] \\
& =\left(\left(S a \cup S^{2} a \cdot a\right)\left(S a \cup S^{2} a \cdot a\right)\right] \subseteq((S a \cup S a)(S a \cup S a)] \\
& =(S a \cdot S a]=(a S \cdot a S] .
\end{aligned}
$$

Hence by using Lemma 4.2, $S$ is strongly regular commutative monoid.

$($ ii $) \Longrightarrow(i):$ It is obvious.

Let $S$ be an ordered $\mathcal{A}^{*} \mathcal{G}^{* *}$-groupoid. From now onward, $R$ (resp. L) will denote any right (resp. left) ideal of $S$; $\langle R\rangle_{a^{2}}$ will denote a right ideal $\left(S a^{2} \cup a^{2}\right]$ of $S$ containing $a^{2}$ and $\langle L\rangle_{a}$ will denote a left ideal $(S a \cup a]$ of $S$ containing $a$; $f_{A}\left(r e s p . g_{B}\right)$ will denote any $D F S$-ideal over $U($ resp. $D F S l$-ideal over $U)$ of $S$ unless otherwise specified.

Theorem 4.6. Let $S$ be an ordered $\mathcal{A}^{*} \mathcal{G}^{* *}$-groupoid. Then $S$ is strongly regular if and only if $\langle R\rangle_{a^{2}} \cap\langle L\rangle_{a}=$ $\left(\langle R\rangle_{a^{2}}^{2}\langle L\rangle_{a}^{2}\right]$ and $\langle R\rangle_{a^{2}}$ is semiprime.

Proof. Necessity: Let $S$ be strongly regular. It is easy to see that $\left(\langle R\rangle_{a^{2}}^{2}\langle L\rangle_{a}^{2}\right] \subseteq\langle R\rangle_{a^{2}} \cap\langle L\rangle_{a}$. Let $a \in$ $\langle R\rangle_{a^{2}} \cap\langle L\rangle_{a}$. Then there exist some $x, y \in S$ such that

$$
\begin{aligned}
a & \leq a x \cdot a y \leq(a x \cdot a y) x \cdot(a x \cdot a y) y=(x \cdot a y)(a x) \cdot(y \cdot a y)(a x) \\
& =(a \cdot x y)(a x) \cdot\left(a y^{2}\right)(a x)=(a \cdot x y)(a x) \cdot(x a)\left(y^{2} a\right) \\
& \in\left(\langle R\rangle_{a^{2}} S \cdot\langle R\rangle_{a^{2}} S\right)\left(S\langle L\rangle_{a} \cdot S\langle L\rangle_{a}\right) \subseteq\langle R\rangle_{a^{2}}^{2}\langle L\rangle_{a}^{2}
\end{aligned}
$$

which shows that $\langle R\rangle_{a^{2}} \cap\langle L\rangle_{a}=\left(\langle R\rangle_{a^{2}}^{2}\langle L\rangle_{a}^{2}\right]$. It is easy to see that $\langle R\rangle_{a^{2}}$ is semiprime. 
Sufficiency: Since $\left(S a^{2} \cup a^{2}\right]$ and $(S a \cup a]$ are the right and left ideals of $S$ containing $a^{2}$ and $a$ respectively. Thus by using given assumption and Lemma 2.1, we get

$$
\begin{aligned}
a & \in\left(S a^{2} \cup a^{2}\right] \cap(S a \cup a]=\left(\left(S a^{2} \cup a^{2}\right]^{2}(S a \cup a]^{2}\right] \\
& =\left(\left(S a^{2} \cup a^{2}\right)\left(S a^{2} \cup a\right) \cdot(S a \cup a)(S a \cup a)\right] \subseteq\left(S\left(S a^{2} \cup a\right) \cdot S(S a \cup a)\right] \\
& =\left(\left(S \cdot S a^{2} \cup S a\right)(S \cdot S a \cup S a)\right]=\left(\left(a^{2} S \cdot S \cup S a\right)(a S \cdot S \cup S a)\right] \\
& =\left(\left(a^{2} S \cdot S \cup S a\right)(a S \cdot S \cup S a)\right]=\left(\left(S a^{2} \cup S a\right)(S a \cup S a)\right] \\
& =\left(\left(a^{2} S \cup S a\right)(S a \cup S a)\right]=((S a \cdot a \cup S a)(S a \cup S a)] \subseteq((S a \cup S a)(S a \cup S a)] \\
& =(S a \cdot S a]=(a S \cdot a S] .
\end{aligned}
$$

This implies that $S$ is strongly regular.

Corollary 4.3. Let $S$ be an ordered $\mathcal{A}^{*} \mathcal{G}^{* *}$-groupoid. Then $S$ is strongly regular if and only if $\langle R\rangle_{a^{2}} \cap\langle L\rangle_{a}=$ $\left(\langle L\rangle_{a}^{2}\langle R\rangle_{a^{2}}^{2}\right]$ and $\langle R\rangle_{a^{2}}$ is semiprime.

Theorem 4.7. Let $S$ be an ordered $\mathcal{A}^{*} \mathcal{G}^{* *}$-groupoid. Then the following conditions are equivalent:

(i) $S$ is strongly regular;

(ii) $\langle R\rangle_{a^{2}} \cap\langle L\rangle_{a}=\left(\langle L\rangle_{a}^{2}\langle R\rangle_{a^{2}}^{2}\right]$ and $\langle R\rangle_{a^{2}}$ is semiprime;

(iii) $R \cap L=\left(L^{2} R^{2}\right]$ and $R$ semiprime;

(iv) $f_{A} \sqcap g_{B}=\left(f_{A} \diamond g_{B}\right) \diamond\left(f_{A} \diamond g_{B}\right)$ and $f_{A}$ is DFS semiprime;

$(v) S$ is strongly regular and $|E|=1,(a=a x \cdot a, \forall a, x \in E)$;

(vi) $S$ is strongly regular and $\emptyset \neq E \subseteq S$ is semilattice.

Proof. $(i) \Longrightarrow(v i)$ : It can be followed from Corollary $4.2(i)$.

$(v i) \Longrightarrow(v)$ : It can be followed from Corollary $4.2(i i)$.

$(v) \Longrightarrow(i v)$ : Let $f_{A}$ and $g_{B}$ be any DFS r-ideal and DFS l-ideal of a strongly regular $S$ over $U$ respectively. From Lemma 3.2, it is easy to show that $\left(f_{A} \diamond g_{B}\right) \diamond\left(f_{A} \diamond g_{B}\right) \sqsubseteq f_{A} \sqcap g_{B}$. Now for $a \in S$, there exist some $x, y \in S$ such that

$$
\begin{aligned}
a & \leq a x \cdot a y \leq(a x \cdot a y) x \cdot(a x \cdot a y) y=(a x \cdot a y) \cdot((a x \cdot a y) x) y \\
& =(a x \cdot a y) \cdot(y x)(a x \cdot a y)=(a x \cdot a y) \cdot(a x)(y x \cdot a y) \\
& =(a x \cdot a y) \cdot(a y \cdot y x)(x a)=(a x \cdot a y) \cdot((y x \cdot y) a)(x a) \\
& =(a x)((y x \cdot y) a) \cdot(a y)(x a)=(a x)(b a) \cdot(a y)(x a), \text { where } y x \cdot y=b .
\end{aligned}
$$


Thus $(a x \cdot b a, a y \cdot x a) \in A_{a}$. Therefore

$$
\begin{array}{r}
\left(\left(f_{A}^{+} \sim g_{B}^{+}\right) \sim\left(f_{A}^{+} \sim g_{B}^{+}\right)\right)(a) \\
=\bigcup_{(a x \cdot b a, a y \cdot x a) \in A_{a}}\left\{\left(f_{A}^{+} \widetilde{\circ} g_{B}^{+}\right)(a x \cdot b a) \cap\left(f_{A}^{+} \widetilde{\circ} g_{B}^{+}\right)(a y \cdot x a)\right\} \\
\supseteq \bigcup_{a x \cdot b a \leq a x \cdot b a}\left\{f_{A}^{+}(a x) \cap g_{B}^{+}(b a)\right\} \cap \bigcup_{a y \cdot x a=a y \cdot x a}\left\{f_{A}^{+}(a y) \cap g_{B}^{+}(x a)\right\} \\
\supseteq f_{A}^{+}(a x) \cap g_{B}^{+}(b a) \cap f_{A}^{+}(a y) \cap g_{B}^{+}(x a) \supseteq f_{A}^{+}(a) \cap g_{B}^{+}(a),
\end{array}
$$

which shows that $\left(f_{A}^{+} \widetilde{\circ} g_{B}^{+}\right) \widetilde{\circ}\left(f_{A}^{+} \widetilde{\circ} g_{B}^{+}\right) \supseteq f_{A}^{+} \cap g_{B}^{+}$. Similarly we can show that $\left(f_{A}^{-} \tilde{\star} g_{B}^{-}\right) \approx\left(f_{A}^{-} \tilde{\star} g_{B}^{-}\right) \subseteq f_{A}^{-} \cup g_{B}^{-}$. Thus $f_{A} \sqcap g_{B} \sqsubseteq\left(f_{A} \diamond g_{B}\right) \diamond\left(f_{A} \diamond g_{B}\right)$. Hence $f_{A} \sqcap g_{B}=\left(f_{A} \diamond g_{B}\right) \diamond\left(f_{A} \diamond g_{B}\right)$. Also by using Lemma 4.6 , $f_{A}$ is $D F S$ semiprime.

$(i v) \Longrightarrow($ iii $)$ : Let $R$ and $L$ be any left and right ideals of $S$. Then by using Lemma $3.1, \mathcal{X}_{R}$ and $\mathcal{X}_{L}$ are the DFS $r$-ideal and DFS l-ideal of $S$ over $U$ respectively. Now by using Lemma 3.4 , we get $\mathcal{X}_{R \cap L}=\mathcal{X}_{R} \sqcap \mathcal{X}_{L}=\left(\mathcal{X}_{R} \diamond \mathcal{X}_{L}\right) \diamond\left(\mathcal{X}_{R} \diamond \mathcal{X}_{L}\right)=\left(\mathcal{X}_{R} \diamond \mathcal{X}_{R}\right) \diamond\left(\mathcal{X}_{L} \diamond \mathcal{X}_{L}\right)=\mathcal{X}_{\left(R^{2}\right]} \diamond \mathcal{X}_{\left(L^{2}\right]}=\mathcal{X}_{\left(R^{2} L^{2}\right]}=\mathcal{X}_{\left(L^{2} R^{2}\right]}$ which implies that $R \cap L=\left(L^{2} R^{2}\right]$.

$($ iii $) \Longrightarrow(i i)$ : It is simple.

$($ ii $) \Longrightarrow(i)$ : It can be followed from Corollary 4.3 .

Theorem 4.8. Let $S$ be an ordered $\mathcal{A}^{*} \mathcal{G}^{* *}$-groupoid. Then the following conditions are equivalent:

(i) $S$ is strongly regular;

(ii) $\langle R\rangle_{a^{2}} \cap\langle L\rangle_{a}=\left(\langle R\rangle_{a^{2}}\langle L\rangle_{a} \cdot\langle R\rangle_{a^{2}}\right]$ and $\langle R\rangle_{a^{2}}$ is semiprime;

(iii) $R \cap L=(R L \cdot R]$ and $R$ is semiprime;

(iv) $f_{A} \sqcap g_{B}=\left(f_{A} \diamond g_{B}\right) \diamond f_{A}$ and $f_{A}$ is DFS semiprime;

(v) $S$ is strongly regular and $|E|=1,(a=a x \cdot a, \forall a, x \in E)$;

(vi) $S$ is strongly regular and $\emptyset \neq E \subseteq S$ is semilattice.

Proof. $(i) \Longrightarrow(v i)$ : It can be followed from Corollary $4.2(i)$.

$(v i) \Longrightarrow(v)$ : It can be followed from Corollary $4.2(i i)$.

$(v) \Longrightarrow(i v)$ : Let $f_{A}$ and $g_{B}$ be any $D F S$ l-ideals of a strongly regular $S$ over $U$. Now for $a \in S$, there exist some $x, y \in S$ such that $a \leq a x \cdot a y \leq a x \cdot(a x \cdot a y) y=((a x \cdot a y) y \cdot x) a=(x y \cdot(a x \cdot a y)) a=(a x \cdot(x y \cdot a y)) a=$ $(a x \cdot(a \cdot(x y) y)) a$. 
Thus $(a x \cdot(a \cdot(x y) y), a) \in A_{a}$. Therefore

$$
\begin{array}{r}
\left(\left(f_{A}^{+} \widetilde{\circ} g_{B}^{+}\right) \widetilde{\circ} f_{A}^{+}\right)(a) \\
=\bigcup_{(a x \cdot(a \cdot(x y) y), a) \in A_{a}}\left\{\left(f_{A}^{+} \tilde{\circ} g_{B}^{+}\right)(a x \cdot(a \cdot(x y) y)) \cap g_{B}^{+}(a)\right\} \\
\supseteq \bigcup_{a x \cdot(a \cdot(x y) y \leq a x \cdot(a \cdot(x y) y}\left\{f_{A}^{+}(a x) \cap g_{B}^{+}(a \cdot(x y) y)\right\} \cap g_{B}^{+}(a) \\
\supseteq f_{A}^{+}(a x) \cap g_{B}^{+}(a \cdot(x y) y) \cap g_{B}^{+}(a) \supseteq f_{A}^{+}(a) \cap g_{B}^{+}(a),
\end{array}
$$

which shows that $\left(f_{A}^{+} \tilde{\circ} g_{B}^{+}\right) \widetilde{\circ} f_{A}^{+} \supseteq f_{A}^{+} \cap g_{B}^{+}$. Similarly we can show that $\left(f_{A}^{-} \tilde{\star} g_{B}^{-}\right) \widetilde{\star} f_{A}^{-} \subseteq f_{A}^{-} \cup g_{B}^{-}$. Thus $\left(f_{A} \diamond g_{B}\right) \diamond f_{A} \sqsupseteq f_{A} \sqcap g_{B}$. By using Lemmas 3.2 and 4.6, it is easy to show that $\left(f_{A} \diamond g_{B}\right) \diamond f_{A} \sqsubseteq f_{A} \sqcap g_{B}$. Thus $f_{A} \sqcap g_{B}=\left(f_{A} \diamond g_{B}\right) \diamond f_{A}$. Also by using Lemma 4.6, $f_{A}$ is $D F S$ semiprime.

$($ iv $) \Longrightarrow($ iii $)$ : Let $R$ and $L$ be any left and right ideals of $S$. Then by Lemma 3.1, $\mathcal{X}_{R}$ and $\mathcal{X}_{L}$ are the $D F S$-ideal and DFS $l$-ideal of $S$ over $U$ respectively. Now by using Lemmas 3.4, 4.1 and 2.1, we get

$$
\mathcal{X}_{R \cap L}=\mathcal{X}_{R} \sqcap \mathcal{X}_{L}=\left(\mathcal{X}_{R} \diamond \mathcal{X}_{L}\right) \diamond \mathcal{X}_{L}=\mathcal{X}_{((R L] \cdot R]}=\mathcal{X}_{(R L \cdot R]},
$$

which shows that $R \cap L=(R L \cdot R]$. Also by using Lemma 3.3, $R$ is semiprime.

$($ iii $) \Longrightarrow($ ii $)$ : It is simple.

$(i i) \Longrightarrow(i)$ : Since $\left(S a^{2} \cup a^{2}\right]$ and $(S a \cup a]$ are the right and left ideals of $S$ containing $a^{2}$ and $a$ respectively.

Thus by using given assumption and Lemma 2.1, we get

$$
\begin{aligned}
a & \in\left(S a^{2} \cup a^{2}\right] \cap(S a \cup a]=\left(\left(S a^{2} \cup a^{2}\right](S a \cup a] \cdot\left(S a^{2} \cup a^{2}\right]\right] \\
& =\left(\left(S a^{2} \cup a^{2}\right)(S a \cup a) \cdot\left(S a^{2} \cup a^{2}\right)\right] \subseteq\left(S(S a \cup a) \cdot\left(S a^{2} \cup a^{2}\right)\right] \\
& =\left(\left(S^{2} a \cup S a\right)\left(S a^{2} \cup a^{2}\right)\right]=\left(\left(S^{2} a \cdot S a^{2}\right) \cup\left(S^{2} a \cdot a^{2}\right) \cup\left(S a \cdot S a^{2}\right) \cup\left(S^{2} a \cdot a^{2}\right)\right] \\
& \subseteq\left(\left(S a \cdot a^{2} S\right) \cup(S a \cdot S a) \cup\left(S a \cdot a^{2} S\right) \cup(S a \cdot S a)\right] \\
& \subseteq((S a \cdot S a) \cup(S a \cdot S a) \cup(S a \cdot S a) \cup(S a \cdot S a)]=(S a \cdot S a]=(a S \cdot a S] .
\end{aligned}
$$

Hence $S$ is strongly regular.

\section{Conclusions}

We have got some interesting and new characterizations which we usually do not find in other algebraic structures. We have considered the following problems in detail:

i) Define and compare DFS left/right ideals of an ordered $\mathcal{A G}$-groupoid and respective examples are provided.

ii) Introduce the concept of an ordered $\mathcal{A}^{*} \mathcal{G}^{* *}$-groupoid and characterize it by using $D F S$ left/right ideals. 
iii) Study the structural properties of a unitary ordered $\mathcal{A G}$-groupoid and ordered $\mathcal{A}^{*} \mathcal{G}^{* *}$-groupoid in terms of its semilattices, strongly regular classes and generated commutative monoids.

This paper generalized the theory of an $\mathcal{A G}$-groupoid in the following ways:

i) In an $\mathcal{A G}$-groupoid (without order) by using the $D F S$-sets.

ii) In an $\mathcal{A G}$-groupoid (with and without order) by using fuzzy sets instead of $D F S$-sets.

Some important issues for future work are:

i) To develop strategies for obtaining more valuable results in related areas.

ii) To apply these notions and results for studying DFS expert sets and applications in decision making problems.

\section{REFERENCES}

[1] M. I. Ali, F. Feng, X. Liu, W. K. Mine and M. Shabir, On some new operations in soft set theory, Comput Math Appl., 57 (2009), 1547-1553.

[2] N. Cagman and S. Enginoglu, FP-soft set theory and its applications, Ann. Fuzzy Math. Inform., 2 (2011), $219-226$.

[3] W. A. Dudek and R. S. Gigon, Congruences on completely inverse AG**-groupoids, Quasigroups and related systems, 20 (2012), 203-209.

[4] W. A. Dudek and R. S. Gigon, Completely inverse AG**-groupoids. Semigroup Forum, 87 (2013), 201-229.

[5] F. Feng, Soft rough sets applied to multicriteria group decision making, Ann. Fuzzy Math. Inform., 2 (2011), 69-80.

[6] F. Feng, Y. B. Jun and X. Zhao, Soft Semirings, Comput. Math. Appl., 56 (2008), 2621-2628.

[7] Y. B. Jun, Soft BCK/BCI-algebras, Comput. Math. Appl., 56 (2008), 1408-1413.

[8] Y. B. Jun and S. S. Ahn, Double-framed soft sets with applications in BCK/BCI-algebras, J. Appl. Math., 2012 , pp. 15.

[9] Y. B. Jun, K. J. Lee and A. Khan, Soft ordered semigroups, Math. Logic Q., 56 (2010), 42-50.

[10] M. A. Kazim and M. Naseeruddin, On almost semigroups, The Alig. Bull. Math., 2(1972), 1-7.

[11] M. Khan, Some studies in AG*-groupoids, Ph. D Thesis, Quaid-i-Azam University, Pakistan, 2008.

[12] A. Khan, T. Asif and Y. B. Jun, Double-framed soft ordered semigroups, submitted.

[13] D. V. Kovkov, V. M. Kolbanov and D. A. Molodtsov, Soft sets theory based optimization, J. Comput. Syst. Sci. Int., 46 (6) (2007), 872-880.

[14] P. K. Maji A. R. Roy and R. Biswas, An application of soft sets in a decision making problem, Comput. Math. Appl., 44 (2002), 1077-1083.

[15] D. Molodtsov, V. Y. Leonov and D. V. Kovkov, Soft sets technique and its application, Nechetkie Sistemy i Myagkie Vychisleniya, 1 (1) (2006), 8-39.

[16] P. Majumdar and S. K. Samanta, Similarity measures of soft sets, New Math. Neutral Comput., 4 (1) (2008), 1-12.

[17] D. Molodtsov, Soft set theory, Comput Math Appl., 37 (1999), 19-31.

[18] Q. Mushtaq and S. M. Yusuf, On LA-semigroups, Alig. Bull. Math., 8(1978), 65-70.

[19] Q. Mushtaq and S. M. Yusuf, On locally associative left almost semigroups, J. Nat. Sci. Math., 19(1979), 57-62.

[20] D. W. Pei and D. Miao, From soft sets to information systems, IEEE International Conference on granular computing, (2005), 617-621.

[21] P. V. Protić and N. Stevanović, AG-test and some general properties of Abel-Grassmann's groupoids, PU. M. A., 4, 6 (1995), 371-383. 
[22] A. R. Roy and P. K. Maji, A fuzzy soft set theoretic approach to decision making problems, J. Comput. Appl. Math., 203 (2007), 412-418.

[23] A. Sezgin, A. O. Atagun and N. Cagman, Soft intersection nearrings with applications, Neural Comput. and Appl., 21 (2011), 221-229.

[24] A. Seizgin and A. O. Atagun, On operations of soft sets, Comput. Math. Appl., 61 (2011), 1457-1467.

[25] N. Stevanović and P. V. Protić, Composition of Abel-Grassmann's 3-bands, Novi Sad, J. Math., 2, 34 (2004), 175-182.

[26] X. Yang, D. Yu, J. Yang, C. Wu, Generalization of soft set theory from crisp to fuzzy case, Fuzzy Inf. Eng., 40 (2007), 345-355.

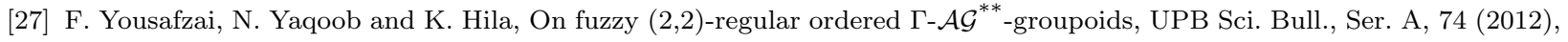
87-104.

[28] F. Yousafzai, A. Khan, V. Amjid and A. Zeb, On fuzzy fully regular ordered AG-groupoids, J. Intell. Fuzzy Syst., 26 (2014), 2973-2982. 